\title{
Carnets
}

Revue électronique d'études françaises de l'APEF

Deuxième série - 9 | 2017

Reconnaissances et légitimité en français

\section{Du bon et du mauvais usage du pseudonyme}

Reconnaissance et délégitimation de Lila dit ça, de Chimo

\section{Cristina Álvares}

\section{OpenEdition}

Journals

Édition électronique

URL : https://journals.openedition.org/carnets/2106

DOI : $10.4000 /$ carnets. 2106

ISSN : 1646-7698

Éditeur

APEF

Référence électronique

Cristina Álvares, « Du bon et du mauvais usage du pseudonyme », Carnets [En ligne], Deuxième série -

9 | 2017, mis en ligne le 31 janvier 2017, consulté le 21 septembre 2021. URL : http://

journals.openedition.org/carnets/2106; DOI : https://doi.org/10.4000/carnets.2106

Ce document a été généré automatiquement le 21 septembre 2021.

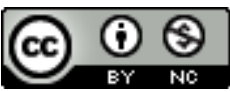

Carnets est mis à disposition selon les termes de la licence Creative Commons - Atribution - Pas d'utilisation commerciale 4.0 International. 


\title{
Du bon et du mauvais usage $\mathrm{du}$ pseudonyme
}

\author{
Reconnaissance et délégitimation de Lila dit ça, de Chimo
}

Cristina Álvares

\section{Beur?}

1 Depuis les années 1980, lorsqu'elle émerge associée à la Marche des Beurs ${ }^{1}$, la littérature produite par les écrivains français nés de parents immigrés du Maghreb fait l'objet d'une revendication de reconnaissance et de légitimation culturelle qui prolonge la revendication politique d'intégration à la société française des populations issues de l'immigration. L'histoire de ce mouvement littéraire est donc celle de la lutte pour une place dans le champ littéraire français moyennant l'investissement du dispositif institutionnel qui y introduit oeuvre et auteur: maisons d'édition, presse et medias audiovisuels, librairies, prix littéraires, universités. Cet appareil de reconnaissance et de légitimation, Michel Laronde l'appelle l'Institution. L'un des instruments de l'Institution est la catégorisation.

2 Dès son émergence, cette littérature a été catégorisée comme littérature beur, arabofrançaise, issue de l'immigration, de banlieue, urbaine ${ }^{2}$, francophone ${ }^{3}$, etc. Créées par les médias, ces catégories ont été adoptées par le discours universitaire (cf. Laronde, 2002 ; Ollson, 2011 ; Puig, 2011 ; Vitali, 2011, 2012 ; Hargreaves, 2014) mais ont été critiquées par les écrivains concernés qui leur reprochent d'enfermer leurs ouvrages dans le déterminisme ethnoculturel. Cela s'applique aussi à la désignation «littérature de banlieue » qui tend, surtout depuis 2005, à remplacer celle de «littérature beur » pour signifier une re-orientation thématique, saisissable dès les années 1990, où le social (l'intégration) l'emporte sur l'identitaire (départenance culturelle). Mais dans la mesure où le milieu social de la banlieue est fortemente ethnicisé et que les écrivains urbains représentent eux-mêmes la continuité entre ces deux périphéries, l'une externe et l'autre interne à la France, que sont les ex-colonies et les cités", la catégorie " de banlieue », loin d'effacer le critère ethnoculturel, le territorialise en leur assignant l'espace périphérique 
des quartiers défavorisés. C'est justement ce déterminisme ethnique et social que ces écrivains contestent, depuis Azouz Begag jusqu'à Mohamed Razane et aux autres écrivains signataires du manifeste Qui fait la France?, au nom d'une approche universaliste de la littérature évaluée selon "des critères communs au commun des écrivains " (Herzoune, 2001: 19) et légitimée par sa valeur esthétique. Or les oeuvres étiquettées «beurs » ou «de banlieue» sont lues moins pour leur qualité littéraire que pour leur valeur documentaire, les auteurs étant perçus comme des ethnographes de banlieue ${ }^{6}$. Il semble donc que la catégorisation de cette littérature produise un effet paradoxal. D'un côté, elle exprime, comme le dit Alec Hargreaves, l'entrée dans le champ littéraire français des minorités post-coloniales, de l'autre elle tient leurs oeuvres à distance, dans les marges de ce champ, les ghettoïse. Par le biais du qualificatif, l'Institution reconnait une littérature qu'elle délégitime dans le même geste comme peu-de-littérature. Aussi Razane demande-t-il: «(...) mais pourquoi chercher un qualificatif? Pourquoi ne pas parler de littérature? ? (apud Vitali, 2012:51). E il ajoute dans un entretien avec Steven Puig :

Je suis un écrivain français qui, comme tout autre écrivain, aspire à l'universalisme. Que je trempe ma plume dans le magma des souffrances de nos territoires en peine ne doit pas ériger mon oeuvre en objet social. C'est avant tout de la littérature, et notre pays, contrairement à l'étranger, peine à le comprendre. "Beur ", " arabe ", «urbaine» sont autant de qualificatifs que je récuse. Je suis et je demeure un écrivain français, certes avec des origines, mais n'est-ce pas là l'histoire de la France? (Puig, 2008: 88).

3 Il faut donc selon Razane dépasser la portée particulariste de la catégorie pour que la reconnaissance littéraire soit intégration à l'identité nationale ${ }^{7}$.

\section{Pseudonyme et aporie}

Dans les années 1990, deux écrivains soi-disant beurs, Paul Smaïl et Chimo, ont eu recours au pseudonyme pour problématiser la catégorie beur et le critère ethnique. Mais tandis que la vraie identité de Smail est connue (c'est Daniel Théron, alias Jacques-Alain Léger), on ne sait toujours pas qui est Chimo. La critique assume que les deux écrivains sont de faux beurs et la discussion tourne autour de la perception du pseudonyme soit comme une imposture soit comme un jeu de masques (Horvath, 2004, Begag, 2006, Varga, 2012) ${ }^{8}$. Ce qui me semble intéressant dans le pseudonyme, c'est qu'il met en lumière le décalage entre auteur-individu-réel et auteur-figure-fictionnelle, c'est-à-dire l'auteur en tant qu'élément du dispositif paratextuel. Un tel décalage dénaturalise la littérature beur, tout en déclenchant la question : « est-ce qu'il faut être beur pour écrire un roman beur ? ${ }^{9}$

Chimo a publié deux romans : Lila dit ça en 1996 et J'ai peur l'année suivante, tous les deux chez Plon ${ }^{10}$. J'ai peur, qui raconte l'échec social et moral du narrateur, n'a pas eu le même impacte que Lila dit ça et l'auteur a quitté la scène littéraire sans avoir découvert son visage. Il n'y a pas de photo de lui, aucune image, il n'a jamais été sur les plateaux. « Il y a des mecs qu'on voit partout à la télé (...) Moi, c'est le contraire. On ne m'a pas vu, on ne me verra pas »11 (1997: 12), sans doute pour cacher son identité ethnique car, comme il le dit plus loin, la tête manifeste l'origine (idem : 218). Lila dit ça a été un succès éditorial ${ }^{12}$, traduit en quinze langues, adapté par le cinéaste franco-libanais Ziad Doueiri en 2005. Un groupe rock québécois s'est fait baptiser Lila dit ça pour rendre hommage à Chimo ${ }^{13}$. C'est dire la projection du roman hors l'Hexagone. En refusant toute exposition médiatique, Chimo s'est vigoureusment détaché des écrivains en quête de visibilité et a montré que la 
médiatisation n'est pas nécessaire au succès commercial et à la diffusion internationale d'une oeuvre. Ce faisant, il a non seulement gardé le mystère de son identité mais il a aussi disjoint l'auteur réel, resté invisible et méconnu, et l'oeuvre, devenue célèbre. Ce qui est encore une façon de contrer l'action des médias audiovisuels qui promeuvent le culte de l'image de l'auteur au détriment de la connaissance de son oeuvre (cf. Soares, 2012).

Lila dit ça est un roman de banlieue qui décrit les conditions matérielles de vie des habitants de la cité du Vieux Chêne: la pauvreté, la saleté, la laideur, les ascenseurs toujours en panne ; ainsi que leur forme de vie : la petite délinquance pratiquée par des jeunes désoeuvrés, le marché noir, les bagarres, les tournantes, la stigmatisation, la désolation, l'ennui. La population est multiethnique et majoritairement de tradition musulmane comme l'indiquent les références à la mosquée, au frère musulman, au Coran, au sacrífice du mouton, bien qu'il y ait aussi quelques rares chrétiens comme la tante béate de Lila : « Les cathos trados comme la tante, une minoritée menacée vu que le Christ il lâche du terrain (...)»(Chimo, 1996: 150). Chimo reprend et développe le motif du béton, dont la matière morte a une portée ontologique qui s'exprime dans l'ennui et le vide d'une vie privée de rêve et d'avenir, une vie coincée :

Les arbres ils sont tout comme nous, plantés là comme des objets sans savoir pourquoi, comme nous sans pouvoir bouger, tu me diras que tous les arbres c'est pareil mais eux c'est pire ils sont dans la mocheté, dans le soleil gris, il leur faut tout faire venir d'ailleurs et puis ils sont tous les mêmes, pas varié du tout comme compagnie, ni buissons ni fleurs because vandalisme, un arbre tous les quinze mètres en ligne droite, ma mère dit que le matin quand elle s'en va de bonne heure s'il fait du vent elle les entend pleurer (idem : 74).

Lila dit ça c'est aussi un Bildungsroman qui raconte le devenir-écrivain de Chimo à travers son initiation au sexe par Lila, une jeune fille blonde d'origine polonaise, dont la beauté fait tache dans le paysage en béton. Lila parle sexe - c'est ça qu'elle dit -, elle verbalise effrontément ses fantasmes, ce qui inscrit ce roman dans le genre érotique à la limite du porno. Le dire impudique de Lila « fait bouger tout ce qui est par là et pourtant elle parle pas fort. Même les arbres qui ont l'air d'être en béton, ils sont touchés quand elle parle " (Chimo, 1996 : 14). Bref, Lila érotise la banlieue ${ }^{14}$. La vie de Chimo est bouleversée par la transfiguration de l'espace qu'opère son dire : «Pour une fois la lumière est venue au Vieux Chêne, un morceau de ciel s'est posé sur nous, pour une fois tu as quelque chose qui est autre chose » (idem : 62). Ce qu'elle lui dit, lui donne à voir (scène du toboggan) et à toucher (scène du vélo) le fait rêver, le fait donc exister et cela d'autant plus qu'elle méprise tous les autres jeunes gens et ne parle qu'à lui seul. Le choc éprouvé par Chimo face à Lila, caucasienne de tradition catholique, n'a rien de culturel, c'est tout simplement sexuel. La conséquence en est qu'il se met à écrire le roman que l'on est en train de lire. Le roman représente son propre processus de production en mettant en avant la dynamique pulsionnelle, activée par le dire-ça, qui pousse et imprègne l'écriture. Avant de rencontrer Lila, Chimo était déjà attiré par l'exercice d'écrire qui lui permettait de s'isoler avec la langue dont il voulait jouir pour échapper à la misère linguistique de son milieu, pour ne pas être « exclu du trésor de la langue » (ibidem : 73). Mais il avait du mal à écrire. Or le dire-ça de Lila débloque l'écriture, la rend fluide, les mots giclent, il jouit d'écrire. Il se compare à une machine qui ne fait qu'enregistrer les paroles de Lila :

(...) je suis là,j'écoute et j'écris, même de plus en plus facile à mesure que je déroule, l'écriture aussi moins secouée, plus la peine de me tordre les cheveux à chercher ce que je vais dire, je suis une machine à enregistrer maintenant, j'enregistre Lila qui cause (ibidem : 29).

Quand elle parle Lila moi je l'écoute bouche ouverte, je comprends même pas ce 
qu'elle dit sur le moment, juste j'enregistre comme j'ai dit j'enregistre tout, c'est beaucoup plus facile après pour moi d'écrie, c'est comme si je déroulais ce ruban et j'ai rien qu'à recopier. Si moi je veux écrire tout seul alors c'est la galère amère, je m'énerve je trouve pas, je me tape sur la tête, au contraire si je la fais parler ça va tout seul, facile facile (...) (ibidem : 138-9).

"Je suis là, j'écoute et j'écris ». Cette formule enchaîne les trois actions sans transition dans une séquence fluide, écrire apparaissant comme le produit immédiat d'écouter. Chimo entretient le mythe d'une écriture passive qui ne ferait que transcrire le dire de Lila, une écriture oralisante et spontanée, irréfléchie, machinale, fondée sur une continuité naturelle entre vécu et fiction. Ce mythe est renforcé par un autre, celui du jeune écrivain de banlieue à demi-scolarisé, qui n'est jamais entré dans une librairie (cf. 1996 : 139), qui n'a pas de références littéraires ${ }^{15}$; mais chez qui s'opère une sorte de miracle de création littéraire évoquant vaguement la tradition romantique du génie créateur et de la muse inspiratrice. Bref, si d'une part le pseudonyme dénaturalise le roman beur en problématisant le critère ethnique, d'autre part le mythe de l'écriture spontanée renaturalise le roman de banlieue. N'est-ce pas cette aporie de la dé-rénaturalisation qu'exprime la métaphore de "la fleur poussée dans le béton des cités " dans le 4e de couverture ? Cette métaphore se nourrit de la contradiction entre la fleur, métaphore du roman, connotant sa valeur esthétique, et le béton, métonymie de la cité. La fleur ne pousse pas dans la matière morte du béton, elle pousse dans la chaleur organique de la terre. Si l'on met l'accent sur le béton, on tendra à interpréter la métaphore comme signifiant que le roman est un produit direct du milieu ethno-socioculturel (il a poussé comme une fleur dans la cité). Si l'on met l'accent sur la fleur, on mettra en valeur la signification non-naturelle de la métaphore : la fleur ne pousse pas dans le béton, le rapport n'est pas direct, le milieu ne produit pas de romans directement et spontanément. Car la littérature c'est l'art du langage.

\section{Esthétique, ethnique}

Organisé autour du pseudonyme, le dispositif paratextuel joue un rôle fondamental dans le jeu entre factuel et fictionnel. Il est composé du 4e de couverture, de l'avertissement de l'éditeur, de la photo de la première page du manuscrit lequel se présente sous la forme de deux cahiers Clairefontaine, et de trois notes pages 14, 70, 170 donnant des renseignements sur le manuscrit ${ }^{16}$ affichant un souci de rigueur philologique. La photo du cahier et les notes produisent un puissant effet de réel mais ne disent rien sur l'auteur réel. Le dispositif paratextuel est composé pour entretenir le mystère de l'identité de l'auteur. Le nom ou prénom Chimo ne dit strictement rien sur son origine ethnique. Quelques indices éparpillés dans le discours conduisent à postuler un écrivain beur mais sans jamais pouvoir l'affirmer avec certitude ${ }^{17}$. Par exemple, lorsqu'il esquisse son portrait : «je suis plutôt du type normal banlieue brun et frisé évidemment et les yeux noirs » (1996: 71). Dans J'ai peur, Chimo narrateur-personnage efface les doutes : il est bel et bien français d'origine maghrébine (cf.997: 222-223). Mais l'invisibilité de l'individu Chimo, tout en brisant le pacte autobiographique, garde le doute ${ }^{18}$. L'auteur paratextuel est français d'origine maghrébine mais l'auteur réel demeure incognito.

L'avertissement de l'éditeur, Olivier Orban, affirme «l'étonnante qualité littéraire du récit", en se demandant si l'auteur serait "un écrivain confirmé ou un jeune (beur) talentueux? Un grand écrivain masqué ou un inconnu surdoué », comme le pose le 4e de 
couverture. Bien que la maison d'édition soit divisée quant à cette question, Olivier Orban avance l'hypotèse d'une mystification. Chimo ne serait pas un jeune écrivain de banlieue. Cette hypothèse relève du préjugé de l'Institution sur la littérature beur évaluée et catégorisée selon le critère ethnique : si c'est beur, alors ce n'est pas littéraire ou c'est peu littéraire ; si c'est littéraire, alors ce n'est pas beur (cf. Laronde, 2002 : 136-7). Aussi en juillet 1996, Olivier Orban écrit-il : « Si demain on me disait que c'est un jeune beur qui l'a écrit, j'en serais le premier étonné, même si ce n'est pas impossible. Mais moi, quando j'ai lu le livre, je me suis tout de suite dit qu'il était l'œuvre d'un écrivain confirmé » (apud Laronde, 2001: 136). Le discours médiatique a renchéri sur ce préjugé que Chimo commente dans J'ai peur :

(...) d'un côté ils disaient Chimo est un écrivain, ça me mettait le coeur dans la bouche, et puis tous ils disaient d'accord c'est bien le livre mais c'est pas de moi c'est un autre, forcément un vieux vicelard qui se cache, même un plié de l'Académie, ou Untel machin, ou alors même Orban l'éditeur, lui que j'ai vu que sur une photo et il a pas l'air à sucer du béton. Comme si moi j'étais un écrivain mais j'avais pas le droit de l'être (1997: 10-11).

Mais là où l'avertissement suggère une imposture (Chimo n'est pas beur/de banlieue), le 4e de couverture, en présentant le roman comme « cette fleur poussée sur le béton des cités ", semble postuler au contraire l'authenticité. On touche là à une zone fort ambiguë de l'ensemble paratextuel. «La fleur poussée sur le béton» signifie que l'auteur du roman, dont la qualité littéraire est reconnue d'emblée (la fleur), est un jeune de banlieue, un beur. Si cela se confirmait, alors le critère esthétique, posé a priori, l'emporterait sur le critère ethnique. Ce serait le moment historique où l'Institution aurait à légitimer l'inclusion dans la littérature française d'un écrivain beur. Mais l'opacité du pseudonyme, en empêchant la révélation de l'identité de Chimo, empêche la légitimation. De plus, tout en assumant que l'auteur est beur/de banlieue, la métaphore de la «fleur poussée sur le béton des cités» entretient le mythe d'une écriture spontanée en continuité avec la parole vivante, mythe sous-jacent à la perception sociologique du roman beur comme témoignage, document, peu-de-littérature. Ce qui est une façon de défaire implicitement (sans en avoir l'air) l'a priori esthétique et de reposer le critère ethnique.

Il semble donc que la divergence entre l'avertissement de l'éditeur et le 4e de couverture - imposture vs authenticité - n'est qu'apparente, les deux pièces fondamentales de l'appareil paratextuel contribuant à fermer la voie de la légitimation à Lila dit ça (ou bien l'auteur n'est pas beur parce que son roman est très bien ou alors l'auteur est beur parce que son roman n'est pas très bien: il y a trop de béton sous la fleur). Bref, le paratexte s'arrange pour annuler la subversion du critère ethnique que le pseudonyme déclenchait dans un premier moment moyennant plusieurs disjonctions (auteur-oeuvre, auteur réelauteur paratextuel). Mais c'est aussi sur le pseudonyme que le critère ethnique revient, dans son opposition au critère esthétique, et avec lui revient la catégorie qui range le roman de Chimo dans les marges de la littérature française. 


\section{BIBLIOGRAPHIE}

ÁLVARES, Cristina (2015). « D’une littérature mal nomée ». Mondes Francophones [disponible le 19 janvier 2016]. <URL : http://mondesfrancophones.com/debats/francophonies-et-theories/dunelitterature-mal-nommee/>.

BEGAG, Azouz (2006). « Imposture and Incompetence: Paul Smal's Vivre me tue ». Research in African Literatures, 37, 1, pp. 55-71<https://muse.jhu.edu/journal/169>.

BLum, Sylvie (2012). « Dans le pays Chimo » in Najib Redouane (éd.). Où en est la littérature 'beur'? Paris : L’Harmattan, pp. 93-105. chImo (1996). Lila dit ça. Paris : Plon.

CHIMO (1997). J'ai peur. Paris : Plon.

DJAVANN, Chahdortt (2009). Ne négociez pas avec le regime iranien. Lettre ouverte aux dirigeants occidentaux. Paris : Flammarion.

HARGREAVES, Alec (2014). « De la littérature 'beur' à la littérature de 'banlieue' : des écrivains en quête de reconnaissance ", Africultures. La Marche en héritage. L'héritage culturel de la Marche pour l'égalité et contre le racisme (1983-2013), pp. 144-149.

HORVATH, Christina (2004). «Les masques de Narcisse : identité(s) problématique(s) dans l'oeuvre de Paul Smaïl » in Bruno Blanckeman, Aline Mura-Brunel et Marc Dambre (éds.). Le roman français au tournant $d u X X I^{e}$ siècle. Paris : Presses de la Sorbonne Nouvelle.

LARONDE, Michel (2001). «L'écrivain postcolonial en France et la manipulation de la figure de l'auteur : Chimo, Paul Smaill, Amhed Zitouni » in Charles Bonn, Najib Redouane, Yvette BenayounSzmidt (éds.). Algérie : nouvelles écritures. Paris : L'Harmattan, pp. 133-147.

LARONDE, Michel (2002). « Prise de parole du roman de la postcolonialité en France : vers une sociocritique du canon littéraire » in Beginning in French Literature, French Literature Series, 29, Amsterdam and Atlanta : Rodopi, pp. 169-180.

OLLSEN, Kenneth (2011). Le discours beur comme positionnement littéraire. Romans et textes autobiographiques français (2005-2006) d'auteurs issus de l'immigration maghrébine. Stockholm: Stockholm University Press.

PUIG, Steve (2008). «Interview avec Mohamed Razane », Expressions maghrébines, 7, 1, pp. 85-92. PUIG, Steve (2011). « Du roman beur au roman urbain : de l'intégration d'Azouz Begag à Désintégration d'Ahmed Djouder » in Vitali (2011), pp. 21-46.

PUIG, Steve (2011a). « Littérature-monde et littérature urbaine : deux manifestes, même combat? ", Nouvelles Francographies, 2, 1, pp. 87-95.

REEK, Laura, (2012). « Lettre ouverte au monde des lettres françaises : Sur ma ligne de Rachid Djaïni » in Vitali (2012), pp. 47-69.

SOARES, Corina (2012). Création littéraire sous contexte médiatisé : l'œuvre de Michel Houellebecq, Amélie Nothomb et Jacques Chessex. Aveiro : Universidade de Aveiro (thèse de doctorat polycopiée).

VARGA, Robert (2012). « Pour une historique de la littérature 'beur' : à l'ombre des phénomènes de culte? Azouz Begag contre Paul Smaill » in Vitali (2012), pp. 71-89. 
VITALI, Llaria (2011). Intrangers. Littérature beur. De l'écriture à la traduction. Louvain la Neuve :

L'Harmattan.

VITALI, Llaria (2012). Intrangers. Post-migration et nouvelles frontières de la littérature beur. Paris :

Academia.

\section{NOTES}

1. De son vrai nom Marche pour l'égalité et contre le racisme.

2. «Urbain » est perçu comme moins stigmatisant que « de banlieue » dans la mesure où le terme n'entraîne pas une sémantique périphérique et marginale.

3. Beaucoup de spécialistes se sont penchés sur l'inadéquation de ces désignations (cf. Vitali, 2012 ; Sebhki, 1999). Des écrivains aussi : Begag, Belghoul, Djaïdani, Razane et beaucoup d'autres. En contestant la catégorie "issus de l'immigration », Razane affirme qu'il préfère "sortis de l'immigration » où il y a une signification de dépassement; d'où le label « littérature de la postmigration » proposée par Vitali. La catégorie « francophonie » est rejetée également puisqu'elle entrave leur intégration dans le champ littéraire national (cf. Álvares, 2015).

4. "L'exclusion sociale est très largement vécue comme un prolongement en France de la domination imposée à leurs parents outre-mer pendant l'époque coloniale » (Hargreaves, 2011 : 3). Ce qui conduit Michel Laronde à les comprendre dans la catégorie des écrivains postcoloniaux. Habiba Sebhki ne le suit pas : «La littérature beur ne peut être considérée comme postcoloniale. Bien que ce terme pose problème à cause de ses nombreuses acceptations selon le rapport au temps, à l'espace ou à toute autre appartenance, je pars du principe néanmoins que pour être postcoloniale, une littérature se positionne dans un rapport d'ex-colonisé-colonisateur. Or, 'le discours produit (par la littérature beur) n'est ni une doléance à l'ancien état colonial ni une complaisance à la vision exotique du Maghreb: c'est une voix active, interpellative et revendicative de la place du citoyen dans la société française'».

5. L'appareil théorique et critique n'utilise pas les mêmes critères pour évaluer la littérature française et la littérature beur. Alors que pour celle-là on considère les formes et les valeurs intrinsèques aux oeuvres, celle-ci est perçue en fonction de l'origine de l'auteur. Aussi les auteurs signataires du manifeste Qui fait la France?, dont la plupart sont d'origine maghrébine, mais pas tous, soulignent qu'ils partagent des idées plutôt que des origines.

6. En 1999, Habiba Sebhki écrivait: "Cette littérature [beur], en effet, est tantôt étiquetée maghrébine, tantôt arabe, tantôt européenne, tantôt étrangère ; elle se trouve aussi répertoriée chez les libraires dans la section 'immigration/racisme'. Ce dernier cas est évidemment une éviction totale du champ littéraire même. Toutes ces étiquettes peuvent expliquer que cette littérature ne trouve, à ma connaissance, aucune place dans les anthologies de littérature française ». Voir aussi Reek, $2012: 49$.

7. Des écrivains comme Chahdortt Djavann ou Brina Svit, qui se situent dans la mouvance du Manifeste Pour une littérature-monde en français, proclament leur appartenance à la littérature sans égard à la nationalité. Djavann écrit : «La seule chose dont je suis sûre, c'est que l'exil est mon essence et l'écriture ma naissance. Je suis née exilée et resterai écrivaine de langue française » (Djavann, 2009 : 43). Par contre, les écrivains beurs situent dans le cadre national leur accès à l'universel littéraire. Ils s'affirment « fils de la France, issus d'ici ». Ils sont français et veulent que leurs œuvres soient reconnues comme littérature française. Et Razane d'inscrire le roman soidisant « beur » dans la tradition du roman réaliste en disant que le père fondateur de ce courant n'est pas Begag mais Zola (Puig, 2008: 88). Pour la perception du rapport entre littérature et nation dans les manifestes Pour une littérature-monde en français et Qui fait la France ?, voir Álvares, 2015. 
8. Begag accuse Smaïl d'imposture (2006); Smaïl adresse la même accusation à Chimo (cf. Horvath, $2004: 323$ ). L'un et l'autre lisent l'oeuvre « en tant que témoignage validé par le nom propre d'un jeune auteur beur (et se sentent) forcément trahis par cette usurpation d'identité » (Horvath, 2004 : 330). La ligne de lecture alternative consiste à faire « abstraction de l'identité de l'auteur (et à juger) ses propos légitimes, sa performance littéraire digne d'intérêt et ses jeux d'identités fort amusants " (ibidem).

9. Le pseudonyme Chimo ne dit strictement rien de l'origine ethnique de l'auteur ni ne renvoie à aucune référence culturelle - contrairement à Smail qui renvoie à Ishmaël, le narrateurprotagoniste de Moby Dick, et au fils d'Abraham et Agar, l'ancêtre des Arabes (cf. Horvath, 2004 : 325).

10. Éditeur prestigieux fondé en 1852 par les frères Plon, dirigé par Olivier Orban, publiant des romans de grands auteurs surtout du $\mathrm{xx}^{\mathrm{e}}$ siècle.

11. Même l'éditeur ne l'a jamais rencontré, le manuscrit lui ayant été remis par un avocat - c'est ce que raconte l'éditeur dans son avertissement et que J'ai peur confirme, à la page 9.

12. Dans la rubrique "Livres" de L'express, on lit dans un article publié le $1^{\mathrm{er}}$ mai 1998 sous le titre « Nos champions à l'exportation » : "Autre best-seller national, Lila dit ça de Chimo (Plon), traduit en 15 langues, a déclenché une véritable guerre des enchères entre quatre éditeurs américains et sept éditeurs anglais ».

13. Il est écrit dans le blog de la bande: «Affamé de mots, Frédéric Christian [l'auteurcompositeur et vocaliste] fait la rencontre impromptue de Lila, ange à l'auréole goudronnée et luminescente, à travers l'écriture désinvolte de Chimo» (https://lila-dit-ca.bandcamp.com/ album/ruelle-palace)

14. Pour une analyse du personnage de Lila, ange et putain, et de son rapport à la condition des jeunes filles en banlieue, voir Blum, 2012.

15. Par contre, Paul Smaïl exhibe sa vaste érudition littéraire.

16. Les notes signalent que le premier cahier commence à l'envers, où finit le premier cahier signalé, des ratures dans les trois dernières pages. Dans l'avertissement, l'éditeur affirme avoir rectifié des erreurs d'orthographe et retouché la ponctuation.

17. Autres indices renvoyant au stéréotype du jeune beur : la terre de Sahel qu'il ne connait pas mais dont il entend parler dans la cité (1997 : 19) ; on va encore à la mosquée (idem : 111) ; le mot du père « je ne suis pas de chez moi » suggère qu'il est un immigré (idem : 147).

18. La critique croit en un faux beur. En parlant de Chimo, Llaria Vitali affirme que "tout contribue à [l'] indiquer comme un 'faux beur'« (Vitali, 2012 : 15). Kenneth ollson assume qu'il n'est pas beur; il aurait caché son identité « un pseudonyme afin de se faire passer pour fils d'immigré » (Ollsen, $2011: 19$ ), ce qui l'amène à l'exclure, ainsi que Smaïl, de son corpus.

\section{RÉSUMÉS}

Dans l'ensemble des littératures migrantes, marginales ou marginalisées en français, la littérature beur, devenue au XXIe siècle littérature de banlieue, constitue une catégorie à mettre en perspective avec cette autre catégorie de littératures migrantes dites francophones. Toutes deux sont périphériques, l'une issue des ex-colonies, l'autre issue des cités. Entre ces deux espaces périphériques, hors et dans la France, il y a une continuité générationnelle (les écrivains de banlieue descendant des immigrés des ex-colonies africaines) reliant le passé colonial à la réalité 
sociale des cités. Notre propos est de placer dans ce contexte Lila dit ça et J'ai peur, romans écrits dans les années soixante-dix par le mystérieux Chimo, écrivain que personne n'a jamais vu. Nous analysons le paratexte et le métatexte de ces romans pour saisir les stratégies fictionnelles que Chimo utilise pour jouer avec le pseudonyme et déjouer certains mécanismes de (dé)légitimation littéraire et culturelle mis en œuvre autour de Lila dit ça.

Amongst the migrant, marginal or marginalized literatures in French, the Beur literature, which became the literature de banlieue in the $21^{\text {st }}$ century, represents a category which has to be put into perspective with this other category of migrant literatures called Francophone literatures. Both are peripheral, one comes from the former colonies; the other one comes from the cités. Between these two peripheral areas, outside and inside France, there is a continuity passed from generation to generation (the writers de banlieue are descendants of immigrants from the former colonies) connecting the colonial legacy with the social reality of the cités. Our purpose is to take Lila dit ça and J'ai peur in this context. They are two novels written in the 70s by mysterious Chimo, a writer that no one has ever seen. We analyze the paratext and the metatext of these novels to understand the fictional strategies that Chimo uses to play with the pseudonyms and to thwart some mechanisms of literary and cultural delegitimation implemented about Lila dit ça.

INDEX

Mots-clés : littérature beur, littérature de banlieue, pseudonyme, légitimation

Keywords : beur literature, literature de banlieue, pseudonym, legitimation

\section{AUTEUR}

CRISTINA ÁLVARES

Un. do Minho

calvares[at]ilch.uminho.pt 